\title{
Holmium Laser Enucleation of the Prostate: Comparison of Immediate Postoperative Outcomes in Patients with and without Antithrombotic Therapy
}

\author{
Conrad V. Bishop ${ }^{a} \quad$ Heath Liddella $^{\mathrm{a}}$ Joseph Ischia ${ }^{\mathrm{a}}$ Eldho Paul ${ }^{\mathrm{b}}$ \\ Sree Appu ${ }^{\mathrm{a}}$ Mark Frydenberg ${ }^{\mathrm{a}, \mathrm{c}}$ Trung Pham ${ }^{\mathrm{a}}$

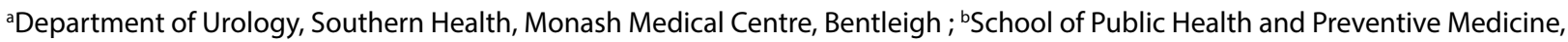 \\ Monash University, Clayton; 'Department of Surgery, Faculty of Medicine, Monash University, Clayton, Australia
}

\section{Key Words}

Prostate - Prostatic hyperplasia • Laser treatment •

Urination disorders • Anticoagulants

\begin{abstract}
Objective: To compare the immediate postoperative outcomes of patients with benign prostatic hyperplasia undergoing Holmium laser enucleation of the prostate (HOLEP) with and without full anticoagulation or antiplatelet therapy at the time of surgery. Materials and Methods: A retrospective review was performed on a series of consecutive patients undergoing HOLEP at our institution by a single surgeon from February 2004 to September 2010. Demographic, surgical, pathological and outcome data were collected. Two cohorts were identified on the basis of antithrombotic therapy at the time of surgery. Patients who continued on aspirin, aspirin/dipyridamole, clopidogrel and warfarin throughout the surgery were included in the antithrombotic cohort. Univariate analysis was performed to determine differences in outcomes between the 2 cohorts. Results: Total 125 consecutive patients underwent HOLEP with 52 patients on antithrombotic therapy at the time of surgery and 73 patients were not on antithrombotic therapy during surgery. Patients in the antithrombotic group were older $(75.1 \pm 7.5$ vs. $71.7 \pm$ 8.3 years; $p=0.02$ ) and had a higher median ASA physical
\end{abstract}

\section{KARGER}

Fax +4161306 1234

E-Mail karger@karger.ch

www.karger.com
(C) 2013 S. Karger AG, Basel

$1015-9770 / 13 / 0071-0028 \$ 26.00 / 0$

Accessible online at:

www.karger.com/cur status ( $3(3-3)$ vs. $2(2-3), p<0.0001)$. The mean operating time and median specimen volume were not significantly different between the 2 cohorts. The median length of stay ( $2(1-3)$ vs. $1(1-2) d, p=0.014)$ was longer in the antithrombotic cohort. The transfusion rate ( 7.7 vs. $0 \%, p=0.028$ ) was predictably higher in the antithrombotic cohort. No patients required re-operation for bleeding. Conclusions: The use of HOLEP in patients on antithrombotic therapy is safe despite the higher surgical risk profile of that particular patient population and the potential increased risk for significant bleeding.

Copyright $\odot 2013$ S. Karger AG, Basel

\section{Introduction}

Symptomatic benign prostatic hyperplasia $(\mathrm{BPH})$ is one of the most common disorders seen in urological practice with an incidence of up to $45.3 \%$ in men aged 65 and over [1]. Transurethral resection of the prostate (TURP) and open prostatectomy have been the gold standards in surgical therapy for symptomatic BPH. Improvements in technology, training and hospital care have seen a steady decline in the rate of significant mor- 
bidity associated with TURP from over $20 \% 30$ years ago to less than $10 \%$ in more recent studies [2-4]. With an ageing population and a high incidence of cardiovascular disease, an increasing number of patients are on antithrombotic therapy [5]. Recent advances in coronary stents have led to a higher proportion of patients on anti-platelet therapy [6]. Cessation of such therapy can lead to stent thrombosis and subsequent acute myocardial infarction with an associated mortality rate of up to $45 \%$. The prevalence of antiplatelet use in urological patients has been identified as a major issue in modern urological surgery $[7,8]$. It is generally accepted that TURP is hazardous on antithrombotic agents, necessitating discontinuation of anticoagulation or antiplatelet therapy for a brief duration to facilitate surgery. This invites the risk of potentially catastrophic thromboembolism and also secondary hemorrhage once antithrombotic therapy is recommenced. This is particularly so in high-risk patients such as those with drug-eluting coronary stents, mechanical cardiac valves, recent pulmonary embolism or atrial fibrillation with prior cerebral ischemia. In addition, TURP is also associated with a hypercoagulable state postoperatively, therefore, further increasing the risk of thrombosis in these patients [9]. Currently, treating these high-risk patients with symptomatic BPH refractory to medical therapy remains a great challenge to the urological surgeon.

Holmium laser enucleation of the prostate (HOLEP) has evolved as an alternative to TURP and open prostatectomy in patients with symptomatic BPH. Recent studies have shown that HOLEP is at least as effective at improving obstructive symptoms with shorter hospitalization and catheter time and importantly less blood loss [10-14]. Two recent studies have supported the safety of HOLEP in anticoagulated patients $[15,16]$. We report our experience with a cohort of patients on antithrombotic therapy i.e. anticoagulant or antiplatelet agents (compared to a cohort of non-antithrombotic patients over the same time frame as a comparison) undergoing HOLEP for symptomatic BPH. To our knowledge, this is the one of the largest studies to date on this increasingly relevant topic as the rate of antithrombotic therapy increases in an aging male population who require surgical intervention for unsuccessful medical therapy for BPH.

\section{Materials and Methods}

A retrospective review was performed on a series of consecutive patients with symptomatic BPH who underwent HOLEP at our institution from February 2004 to September 2010. All procedures were performed by a single surgeon (T.P.). Demographic, clinical, surgical and outcome data were collected. Demographic data included the patient's age and ASA physical status score. The ASA score was determined by the attending anaesthetist. Clinical data included the indication for surgery, use of antithrombotic therapy before or during surgery as well as the indication and type of antithrombotic therapy used. Preoperative evaluation of patients included a history and examination including a digital rectal examination, and when indicated a prostate-specific antigen level. Preoperative international normalized ratio (INR) of less than 4.0 was required. Surgical data included the operative time as well as the volume of specimen. The operative time included the laser and the morcellation time. Outcome data included length of hospital stay, success of initial trial of void (TOV), need for blood transfusion, and return to theatre. The purpose of this study was to evaluate immediate postoperative outcome with respect to the use of anticoagulation, and therefore long-term outcome data such as international prostate symptom score and Qmax are not reported here.

Two cohorts were identified for comparison. The first cohort represents patients not on antithrombotic therapy at the time of surgery. This included patients naïve to antithrombotic therapy as well as those deemed low risk for thromboembolism, discontinuing therapy prior to surgery. There were also a small number of highrisk patients in this cohort who discontinued oral anticoagulants but were bridged with low molecular weight heparin (LMWH). The LMWH was ceased at least 12 hours prior to surgery allowing for normal coagulation parameters. The second cohort included patients deliberately remaining on oral anticoagulant or antiplatelet agents for the procedure. This mainly consisted of patients at high-risk of thromboembolism. Antithrombotics used by patients in this study included the antiplatelet agents aspirin 100-150 mg daily, clopidogrel $75 \mathrm{mg}$ daily, or dipyridamole $200 \mathrm{mg}$ /aspirin 25 $\mathrm{mg}$ twice daily; or the coumarin derivative, warfarin. There were no patients with non-pharmacological bleeding disorders in this series.

HOLEP was performed using a 100W Ho:YAG VersaPulse ${ }^{\circledR}$ Power Suite ${ }^{\mathrm{TM}}$ laser source (Lumenis ${ }^{\circledR}$, Santa Clara, California) with a 550 micron SlimLine ${ }^{\mathrm{TM}}$ end firing laser fiber. This was delivered via a continuous flow 26 Fr resectoscope sheath with bridge and a $5 \mathrm{Fr}$ ureteric catheter to stabilize the fiber. Saline $(0.9 \%)$ was used as the irrigation fluid. Morcellation was performed via a rigid nephroscope using a VersaCut ${ }^{\mathrm{TM}}$ endoscopic tissue morcellator (Lumenis ${ }^{\circledR}$, Yokneam, Israel). A 22 Fr 3-way catheter was inserted after the procedure with or without continuous saline irrigation. Non-anticoagulated patients routinely underwent TOV on the first day postoperatively. A more conservative approach was taken with antithrombotic patients, most undergoing TOV on the second postoperative day.

All data were analyzed using SAS software version 9.1 (SAS Institute Inc., Cary, NC, USA). A comparison between the groups (Antithrombotic vs. Non-antithrombotic) was performed using the student t-test for parametric continuous variables or the Mann-Whitney U test for ordinal variables and chi-square or Fisher's exact test for categorical variables. Continuous variables are reported as mean \pm SD and categorical variables as number (percentage). Ordinal data are reported as median with an inter-quartile range. A two-sided $p$ value of 0.05 was considered statistically significant. 
Table 1. Patient and surgical characteristics

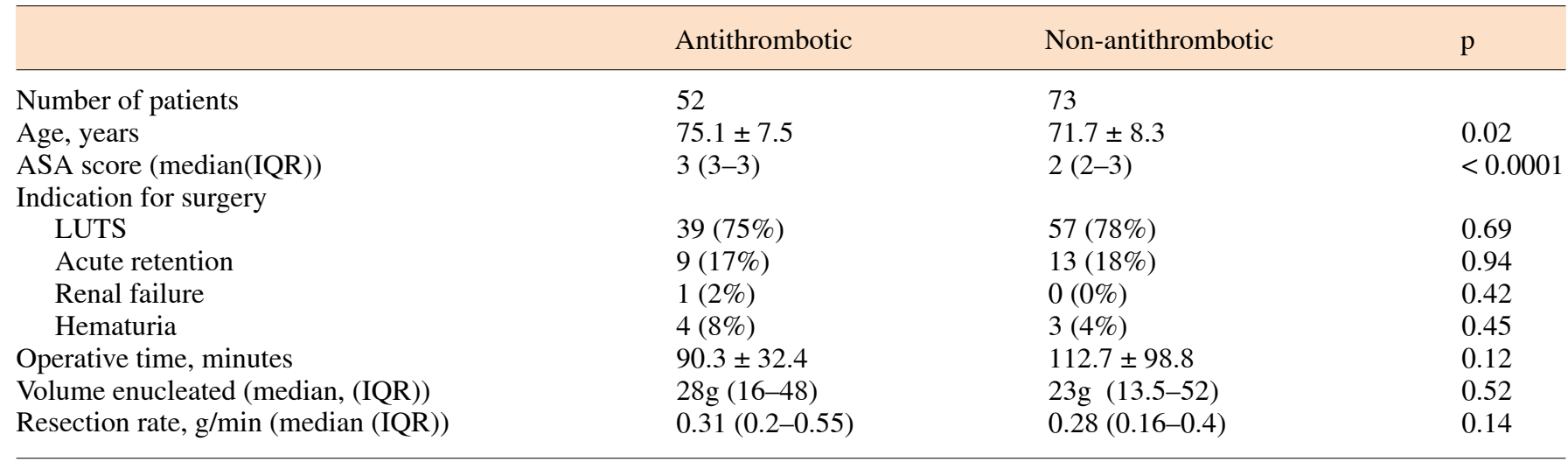

Table 2. Indications for anticoagulation

\begin{tabular}{lc}
\hline Indication & Patients, $\mathrm{n}$ \\
\hline Prosthetic valve & 12 \\
Atrial fibrillation & 9 \\
Coronary stent & 9 \\
Previous stroke/TIA & 8 \\
Unstable angina & 7 \\
DVT/PE & 5 \\
Cardiomyopathy & 1 \\
Mesenteric infarct & 1
\end{tabular}

TIA = Transient ischemic attack; DVT = deep vein thrombi; $\mathrm{PE}=$ pulmonary embolism.

\section{Result}

Total 125 patients underwent HOLEP over the duration of the study, in which 52 patients (41.6\%) remained on oral antithrombotic agents for the procedure and 73 patients (58.4\%) were not on antithrombotic agents at the time of surgery and constituted the comparison cohort. This included patients who ceased antithrombotic agents for an adequate period of time prior to surgery. The patient and surgical characteristics are given in table 1 . The mean age was significantly higher in the antithrombotic cohort, which would be compatible with their poorer health. This was confirmed with the antithrombotic cohort having significantly higher ASA scores. The mean operative time was shorter in the antithrombotic patients, but this result was not statistically significant. There was no significant difference in the volume resected between the 2 cohorts.

Of the 52 patients that remained on antithrombotic agents during the HOLEP, 11 patients were on aspirin, 3 patients on dipyridamole/aspirin, 16 patients on clopidogrel and 22 patients on warfarin. The indications for antithrombotic therapy are summarized in table 2 . The mean INR of the warfarinised patients was 2.61 (SD 0.66) at the time of surgery. The INR range was 1.7 to 3.9.

The immediate postoperative data is summarized in table 3. Four patients $(7.7 \%)$ in the antithrombotic group required blood transfusion postoperatively compared with no patients in the non-antithrombotic cohort ( $\mathrm{p}=$ 0.028 ). Antithombotic use and requirement for transfusion data is summarized in table 4 . Two patients in the non-anticoagulated cohort required a return to theatre. This was due to a delayed small intraperitoneal bladder perforation 2 days after the surgery from the morcellation-associated injury requiring surgical repair. This was not associated with postoperative bleeding. While there was a trend for the antithrombotic group of patients to be more likely to fail their initial TOV, this failed to reach statistical significance in this study $(\mathrm{p}=0.07)$. All of these patients successfully passed a second TOV within 6 weeks of the operation. It is standard practice to remove the catheter on the second post-operative day in the patients on antithrombotic agents but within 24 hours in the non-antithrombotic cohort. There were no thromboembolic events in this series.

Twenty-five men had greater than $60 \mathrm{~g}$ of prostate tissue enucleated which would be a rough estimate of the maximum volume removed during a large TURP. Of these men, 19 had pre- and post-operation hemoglobin 
Table 3. Immediate postoperative data

\begin{tabular}{llll}
\hline & Antithrombotic & Non-antithrombotic & p \\
\hline Patients, n & 52 & 73 & 0.028 \\
Blood transfusion & $4(7.7 \%)$ & $2(2.7 \%)$ & 0.51 \\
Return to theatre & $0(0.0 \%)$ & $5(6.9 \%)$ & 0.07 \\
Failed TOV & $9(17.3 \%)$ & $1(1-2)$ & 0.014 \\
Median (IQR) length of stay, d & $2(1-3)$ & \\
\hline
\end{tabular}

Table 4. Antithrombotic use and associated transfusions

\begin{tabular}{llc}
\hline Antithrombotic & $\mathrm{n}$ & Transfusion \\
\hline Aspirin & 0 & 11 \\
Dipyridamole/Aspirin & 1 & 3 \\
Clopidogrel & 1 & 16 \\
Warfarin & 2 & 22 \\
Total & 4 & 52 \\
\hline
\end{tabular}

values collected as detailed in table 5 . In these men, the median $\mathrm{Hb}$ drop was $15 \mathrm{~g} / \mathrm{l}$ (interquartile range (IQR): 6 to 20). One of these men required a blood transfusion post-operatively. He remained on dipyridamole/aspirin peri-operatively and had $127 \mathrm{~g}$ of prostate tissue enucleated.

\section{Discussion}

The management of antithrombotic patients with symptomatic BPH refractory to medical therapy with alpha antagonists or 5-alpha reductase inhibitors is an increasingly difficult issue. There are effectively 2 categories of oral antithrombotic agents in the general population - the anticoagulant warfarin and antiplatelet agents - and ceasing these agents for safe surgery can be complicated. In the case of warfarin, returning to a normal coagulable state is unpredictable and new guidelines define patients in chronic atrial fibrillation at high risk for stroke [17] who need cover with heparin (usually LMWH) in the peri-operative period. The development of drug-eluting stents for coronary artery disease has introduced this concept of an 'obligatory' period of extremely high risk for thrombosis for ceasing antiplatelet agents which can be up to 12 months [7].

HOLEP: Comparison of Immediate Postoperative Outcomes in Patients with and wthout Antithrombotic Therapy
Table 5. Pre- and post-operation hemoglobin values in those with greater than $60 \mathrm{~g}$ prostate tissue enucleated

\begin{tabular}{ll}
\hline & Antithrombotic $\mathrm{n}=19(25)$ \\
\hline Mean pre-operative $\mathrm{Hb}$ & 142 \\
Mean post-operative $\mathrm{Hb}$ & 126 \\
Mean difference & 16 \\
Median (IQR) Hb change & $15(6-20)$ \\
\hline
\end{tabular}

The correlation between antithrombotic therapy use with age and comorbidity was borne out in our series with the antithrombotic patients being older and having higher ASA physical status scores than the control cohort of non-antithrombotic men. The indications for surgery were similar in both cohorts. As the antithrombotic patients were older and less fit, we expected to see higher rates of urinary retention and hematuria in this cohort although this was not observed in the results and were similar to other studies $[13,15,16,18]$.

In a courageous attempt to determine if oral anticoagulation could be continued during surgery, Parr et al. [19] performed TURPs on 12 fully anticoagulated patients. The authors experienced troublesome bleeding in 50\% of cases requiring immediate reversal of anticoagulation with fresh frozen plasma. Four men (33\%) required additional transfusion of red blood cells. Clearly this is not an acceptable result now that safer alternatives are available.

Gilling et al. [10] published the first large series of HOLEP in 1996. The authors presented 84 non-anticoagulated patients undergoing the procedure with excellent long-term results that compare well to any TURP series. They noted the procedure was relatively bloodless, a characteristic ideal for later use on anticoagulated patients. Kuntz et al. [11] published a series of 200 non-anticoagulated patients with urodynamically proven

Curr Urol 2013;7:28-33 
obstruction randomized to TURP or HOLEP. The hemostatic properties of the holmium laser were again noted with a lesser drop in hemoglobin in the HOLEP group $(1.3 \pm 1.0$ vs. $1.8 \pm 1.0 \mathrm{~g} / \mathrm{dl}, \mathrm{p}=0.01)$. There were no transfusions in the HOLEP group and 2 in the TURP group. After 12 months follow-up, HOLEP was superior in reduction of AUA scores and PVR volumes. The increase in Qmax was similar in both groups. A recent meta-analysis has confirmed that HOLEP has at least comparable efficacy to TURP at relieving symptoms due to $\mathrm{BPH}$ [18]. A current review article also concludes that HOLEP offers favorable and durable outcomes for any prostate size with low early and late morbidity [20].

In antithrombotic patients, vaporization of the prostate with KTP laser has been reported with great success [21, 22]. Reich et al. [21] reported a series of 66 patients with an ASA 3 undergoing KTP prostate vaporization. Sixteen of these patients were on coumarin derivatives and 10 were on platelet inhibitors. None required blood transfusion or reoperation for bleeding. Sandhu et al. [22] similarly reported a series of 26 anticoagulated patients undergoing KTP prostate vaporization. This included 14 patients on aspirin, eight on coumarin derivatives and two on clopidogrel. The patients on coumarin derivatives ceased their medication 2 days prior to surgery and recommenced on the first postoperative day. Again, no patients required blood transfusion or reoperation for bleeding.

In the current study, we report our experience of 52 patients on antithrombotic therapy undergoing HOLEP for symptomatic $\mathrm{BPH}$ and compared this to a control cohort of patients who were not on antithrombotic therapy at the time of surgery. Four men $(7.7 \%)$ in the antithrombotic group required a blood transfusion compared to none in the control group. Of those who required a transfusion, 1 patient was on clopidogrel and received 2 units of packed red blood cells (pRBC), 2 patients were on warfarin (INR 2.9 and 3.9) and received 2 and 4 units of pRBC, respectively, and 1 patient was on the aspirin/ dipyridamole combination and received 2 units pRBC. There were no returns to theatre in the antithrombotic group. To our knowledge, this is the largest study on HOLEP in men on antithrombotic medications. Elzayat et al. [15] reported their experience with HOLEP on 83 men requiring long-term anticoagulation. However, only 14 underwent HOLEP while fully anticoagulated, the remainder withdrew from their medication prior to surgery. The overall transfusion rate in this series was $8.4 \%$. Two of $14(14.2 \%)$ patients who had been fully anticoagulated during surgery required a transfusion. An additional patient on clopidogrel received a platelet transfusion for bleeding. Our transfusion rate of $7.7 \%$ in antithrombotic patients compares favourably. Furthermore, the mean INR in the Elzayat study was 2.0 compared with 2.6 in the current study.

In another study, Tyson et al. [16] reported 38 HOLEPs performed on patients on antithrombotic therapy and $25(66 \%)$ of these patients were only on aspirin compared to $11(21 \%)$ in the current study. Today, many urologists are happy to perform TURP with careful attention to hemostasis with patients on aspirin. The current study is unique in that includes a large number of men on dipyridamole/aspirin (3 men), warfarin (22 men) and probably most significantly, clopidogrel (16 men). Tyson et al. [16] reported that none of their 13 patients on warfarin required blood transfusion. However, in their study, the mean INR was 1.5 with only 2 patients having INRs over 2.0 compared to our mean INR of 2.6 , which may explain our higher transfusion rate of $7.7 \%$.

The operating times were similar in both groups, with comparable prostate sizes reflected by the specimen volumes. It should be noted that the median prostate volumes enucleated of 23 and $28 \mathrm{~g}$ in the control and antithrombotic groups, respectively, appears quite low compared with some contemporary series. However, the upper limit of the interquartile ranges is 48 and $52 \mathrm{~g}$ in each group signifying that $25 \%$ of the prostates enucleated were larger than these values and highlighting some of the very large prostates that were treated. While one may anticipate HOLEP in the anticoagulated patient to be more technically difficult and time consuming, the excellent hemostatic properties of the holmium laser render little difference in operating difficulty when compared to non-anticoagulated patients. In our experience, the surgeon (T.P.) performed an anatomical enucleation and did not feel he had to adjust his operative approach for anticoagulated patients.

Retrospective analyses are an inferior research tool to a well-constructed randomized trial. After the convincing results of Parr et al. [19], a trial comparing the gold standard TURP to HOLEP on anticoagulated patients with symptomatic BPH will never be attempted. The current study has demonstrated that HOLEP in high-risk patients on full antithrombotic therapy is safe and the early post-operative results are comparable to non-antithrombotic patients undergoing TURP $[2,11,13,18]$. As previously mentioned, photoselective vaporization of the prostate with the Greenlight laser is another option for surgically treating men on antithrombotic therapy with symptomatic BPH. Recent reviews have confirmed that 
photoselective vaporization of the prostate also has excellent hemostatic properties in these patients and the 2 modalities are probably equivalent in smaller prostates (up to $60 \mathrm{~g}$ ). However, in larger prostates HOLEP is probably superior in safety, efficacy and time efficiency $[14,23]$.

\section{Conclusions}

The current study demonstrates HOLEP is a safe and effective procedure for fully antithrombotic patients with symptomatic BPH refractory to medical therapy. However, it is worth noting that patients should be taken off antithrombotic therapy whenever possible prior to surgery. With the current increasing number of high-risk men who are on antithrombotic therapy requiring surgical intervention of BPH, HOLEP is one of the best options which the urologic surgeon can offer.

\section{References}

1 Kristal AR, Arnold KB, Schenk JM, Neuhouser ML, Weiss N, Goodman P, Antvelink CM, Penson DF, Thompson IM: Race/ethnicity, obesity, health related behaviors and the risk of symptomatic benign prostatic hyperplasia: results from the prostate cancer prevention trial. J Urol 2007;177:1395-1400.

\2 Borboroglu PG, Kane CJ, Ward JF, Roberts JL, Sands JP: Immediate and postoperative complications of transurethral prostatectomy in the 1990s. J Urol 1999;162:1307-1310.

-3 Rassweiler J, Teber D, Kuntz R, Hofmann $\mathrm{R}$ : Complications of transurethral resection of the prostate (TURP)--incidence, management, and prevention. Eur Urol 2006;50:969979.

4 Mebust WK, Holtgrewe HL, Cockett AT, Peters PC: Transurethral prostatectomy: immediate and postoperative complications. A cooperative study of 13 participating institutions evaluating 3,885 patients. J Urol 1989; 141:243-247.

5 DeWilde S, Carey IM, Emmas C, Richards N, Cook DG: Trends in the prevalence of diagnosed atrial fibrillation, its treatment with anticoagulation and predictors of such treatment in UK primary care. Heart 2006;92: 1064-1070.

6 Trikalinos TA, Alsheikh-Ali AA, Tatsioni A, Nallamothu BK, Kent DM: Percutaneous coronary interventions for non-acute coronary artery disease: a quantitative 20-year synopsis and a network meta-analysis. Lancet 2009;373:911-918.

7 Moussa ID, Colombo A: Antiplatelet therapy discontinuation following drug-eluting stent placement: dangers, reasons, and management recommendations. Catheter Cardiovasc Interv 2009;74:1047-1054.
8 Eberli D, Chassot PG, Sulser T, Samama CM, Mantz J, Delabays A, Spahn DR: Urological surgery and antiplatelet drugs after cardiac and cerebrovascular accidents. J Urol 2010; 183:2128-2136.

$\checkmark 9$ Bell CR, Murdock PJ, Pasi KJ, Morgan RJ: Thrombotic risk factors associated with transurethral prostatectomy. BJU Int 1999;83: 984-989.

10 Gilling PJ, Cass CB, Cresswell MD, Fraundorfer MR: Holmium laser resection of the prostate: preliminary results of a new method for the treatment of benign prostatic hyperplasia. Urology 1996;47:48-51.

$\checkmark 11$ Kuntz RM, Ahyai S, Lehrich K, Fayad A: Transurethral holmium laser enucleation of the prostate versus transurethral electrocautery resection of the prostate: a randomized prospective trial in 200 patients. J Urol 2004;172:1012-1016.

12 Gupta N, Sivaramakrishna, Kumar R, Dogra PN, Seth A: Comparison of standard transurethral resection, transurethral vapour resection and holmium laser enucleation of the prostate for managing benign prostatic hyperplasia of $>40$ g. BJU Int 2006;97:85-89.

-13 Tan AH, Gilling PJ, Kennett KM, Frampton C, Westenberg AM, Fraundorfer MR: A randomized trial comparing holmium laser enucleation of the prostate with transurethral resection of the prostate for the treatment of bladder outlet obstruction secondary to benign prostatic hyperplasia in large glands (40 to 200 grams). J Urol 2003;170:1270-1274.

14 Descazeaud A, Robert G, Azzousi AR, Ballereau C, Lukacs B, Haillot O, Dumonceau O, Devonec M, Fourmarier M, Saussine C, de la Taille A: Laser treatment of benign prostatic hyperplasia in patients on oral anticoagulant therapy: a review. BJU Int 2009;103:1162-1165.
15 Elzayat E, Habib E, Elhilali M: Holmium laser enucleation of the prostate in patients on anticoagulant therapy or with bleeding disorders. J Urol 2006;175:1428-1432.

16 Tyson MD, Lerner LB: Safety of holmium laser enucleation of the prostate in anticoagulated patients. J Endourol 2009;23:13431346.

17 Antithrombotic Therapy and Prevention of Thrombosis, ed 9: American College of Chest Physicians Evidence-Based Clinical Practice Guidelines.

18 Ahyai SA, Gilling P, Kaplan SA, Kuntz RM, Madersbacher S, Montorsi F, Speakman MJ, Stief CG: Meta-analysis of functional outcomes and complications following transurethral procedures for lower urinary tract symptoms resulting from benign prostatic enlargement. Eur Urol 2010;58:384-397.

19 Parr NJ, Loh CS, Desmond AD: Transurethral resection of the prostate and bladder tumour without withdrawal of warfarin therapy. Br J Urol 1989;64:623-625.

20 Gravas S, Bachmann A, Reich O, Roehrborn CG, Gilling PJ, De La Rosette J: Critical review of lasers in benign prostatic hyperplasia (BPH). BJU Int 2011;107:1030-1043.

21 Reich O, Bachmann A, Siebels M, Hofstetter A, Stief CG, Sulser T: High power $(80 \mathrm{~W})$ potassium-titanyl-phosphate laser vaporization of the prostate in 66 high risk patients. J Urol 2005;173:158-160.

-22 Sandhu JS, Ng CK, Gonzalez RR, Kaplan SA, Te AE: Photoselective laser vaporization prostatectomy in men receiving anticoagulants. J Endourol 2005;19:1196-1198.

23 Rieken M, Ebinger Mundorff N, Bonkat G, Wyler S, Bachmann A: Complications of laser prostatectomy: a review of recent data. World J Urol 2010;28:53-62. 